Article

\title{
Conservation Laws of Discrete Evolution Equations by Symmetries and Adjoint Symmetries
}

\author{
Wen-Xiu Ma ${ }^{1,2}$ \\ ${ }^{1}$ College of Mathematics and Physics, Shanghai University of Electric Power, Shanghai 200090, China \\ 2 Department of Mathematics and Statistics, University of South Florida, Tampa, FL 33620, USA; \\ E-Mail: mawx@cas.usf.edu; Tel.: +1-813-9749563; Fax: +1-813-9742700
}

Academic Editor: Roman M. Cherniha

Received: 24 April 2015 / Accepted: 20 May 2015 / Published: 22 May 2015

\begin{abstract}
A direct approach is proposed for constructing conservation laws of discrete evolution equations, regardless of the existence of a Lagrangian. The approach utilizes pairs of symmetries and adjoint symmetries, in which adjoint symmetries make up for the disadvantage of non-Lagrangian structures in presenting a correspondence between symmetries and conservation laws. Applications are made for the construction of conservation laws of the Volterra lattice equation.
\end{abstract}

Keywords: symmetry; adjoint symmetry; conservation law

\section{Introduction}

Noether's theorem tells us that a symmetry of a differential equation leads to a conservation law of the same equation, if the equation is derived from a Lagrangian, namely, it has a Lagrangian formulation [1,2]. The Lagrangian formulation of the equation is essential for presenting conservation laws from symmetries, and many physically important examples can be found in [1-4]. A natural question arises whether there is any correspondence between symmetries and conservation laws for differential equations not derivable from any Lagrangian. We would, in this paper, like to show that it is possible to give a positive answer to the above question if we adopt adjoint symmetries. More precisely, we want to exhibit that using symmetries and adjoint symmetries together can lead to conservation laws for both Lagrangian and non-Lagrangian equations.

A good attempt to use adjoint symmetries in computing conservation laws of differential equations was made in [5], and the approach utilizes adjoint symmetries, in which an adjoint invariance condition 
equivalently requires the existence of an adjoint symmetry. More generally, nonlinear self-adjointness was introduced on the basis of adjoint systems and successfully applied to construction of conservation laws of differential equations [6,7]. In that theory, the nonlinear self-adjointness means that the second set of dependent variables in an adjoint system stands for an adjoint symmetry.

We would like to consider regular differential-difference equations, and so, they can be written as evolution equations. Equations of this type contain difference equations, since any difference equation can be considered as a stationary equation of discrete evolution equations. We will utilize pairs of symmetries and adjoint symmetries to present a direct formula for constructing conservation laws, and thus conserved densities, for evolution equations. Our approach will be used to compute conservation laws for the Volterra lattice equation. The general theory justifies that symmetries really reflect conservation laws. However, the adoption of adjoint symmetries has not attracted much attention within the mathematical physics community. It is expected that our findings could stimulate to expose more mathematical properties of adjoint symmetries as well as develop efficient algorithms for computing adjoint symmetries.

The paper is structured as follows. In Section 2, a general theory will be formulated for constructing conservation laws and thus conserved densities from symmetries and adjoint symmetries for discrete evolution equations. In Section 3, an example will be analyzed, along with new conserved densities. Finally in Section 4, a few of concluding remarks will be given with some discussion.

\section{General Theory}

We will use a plain language to formulate the framework of our results on the correspondence between conservation laws and pairs of symmetries and adjoint symmetries.

Let the potential vector $u$ be defined by

$$
u=\left(u^{1}, \cdots, u^{q}\right)^{T}, u^{i}=u^{i}(n, t), 1 \leq i \leq q, n=\left(n_{1}, \cdots, n_{p}\right) \in \mathbb{Z}^{p}, t \in \mathbb{R},
$$

and $E_{i}, 1 \leq i \leq p$, denote the shift operators for the variables $n_{i}, 1 \leq i \leq p$, i.e.,

$$
\left(E_{i} u\right)(n)=u\left(n_{1}, \cdots, n_{i-1}, n_{i}+1, n_{i+1}, \cdots, n_{p}\right), n=\left(n_{1}, \cdots, n_{p}\right) \in \mathbb{Z}^{p}, 1 \leq i \leq p .
$$

we introduce

$$
E^{\alpha}=E_{1}^{\alpha_{1}} \cdots E_{p}^{\alpha_{p}}, E^{\alpha} u=\left(E^{\alpha} u^{1}, \cdots, E^{\alpha} u^{q}\right)=\left(u_{\alpha}^{1}, \cdots, u_{\alpha}^{q}\right),\left(E^{\alpha} u^{i}\right)(n)=u^{i}(n+\alpha),
$$

where $\alpha=\left(\alpha_{1}, \cdots, \alpha_{p}\right), n=\left(n_{1}, \cdots, n_{p}\right) \in \mathbb{Z}^{p}$, and $n+\alpha=\left(n_{1}+\alpha_{1}, \cdots, n_{p}+\alpha_{p}\right)$. We denote by $\mathcal{A}$ and $\mathcal{B}$ the space of all local $C^{\infty}$ functions in $n, t, u$ and $E^{\alpha} u$ to some finite order $\alpha$, and the space of all $C^{\infty}$ functions in $n, t, u$ and $E^{\alpha} u$ to some finite order $\alpha$. Moreover, we assume that $\mathcal{A}^{r}$ and $\mathcal{B}^{r}$ denote the $r$-th order tensor products of $\mathcal{A}$ and $\mathcal{B}$, respectively, i.e.,

$$
\mathcal{A}^{r}=\underbrace{\mathcal{A} \otimes \cdots \otimes \mathcal{A}}_{r}, \mathcal{B}^{r}=\underbrace{\mathcal{B} \otimes \cdots \otimes \mathcal{B}}_{r}
$$

The locality here means that for a function $f(u)$ in $\mathcal{A}$, any value $(f(u))(n)$ is completely determined by the values of $u$ at finitely many points $n \in \mathbb{Z}^{p}$. The space $\mathcal{A}$ contains functions of polynomial 
type, $P\left(u, E u, \cdots, E^{\alpha} u\right)$, where $P$ is a polynomial in its variables, and the space $\mathcal{B}$ contains non-local functions:

$$
(f(u))(n)=\sum_{|\alpha| \leq m} \sum_{k \geq n}\left(E^{\alpha} u\right)(k), m \geq 1,|\alpha|=\left|\alpha_{1}\right|+\cdots+\left|\alpha_{p}\right| .
$$

For a local vector function $X=X(u)=\left(X_{1}, \cdots, X_{r}\right)^{T} \in \mathcal{A}^{r}$, we can compute its Gateaux derivative operator as follows:

$$
X^{\prime}=X^{\prime}(u)=\left(V_{j}\left(X_{i}\right)\right)_{r \times q}=\left[\begin{array}{cccc}
V_{1}\left(X_{1}\right) & V_{2}\left(X_{1}\right) & \cdots & V_{q}\left(X_{1}\right) \\
V_{1}\left(X_{2}\right) & V_{2}\left(X_{2}\right) & \cdots & V_{q}\left(X_{2}\right) \\
\vdots & \vdots & \ddots & \vdots \\
V_{1}\left(X_{r}\right) & V_{2}\left(X_{r}\right) & \cdots & V_{q}\left(X_{r}\right)
\end{array}\right], V_{i}\left(X_{j}\right)=\sum_{\alpha \in \mathbb{Z}^{p}} \frac{\partial X_{j}}{\partial u_{\alpha}^{i}} E^{\alpha}
$$

and its (formal) adjoint operator

$$
X^{\dagger \dagger}=X^{\dagger \dagger}(u)=\left(V_{i}^{\dagger}\left(X_{j}\right)\right)_{q \times r}, V_{i}^{\dagger}\left(X_{j}\right)=\sum_{\alpha \in \mathbb{Z}^{p}} E^{-\alpha} \frac{\partial X_{j}}{\partial u_{\alpha}^{i}},
$$

where the action of the operator $E^{-\alpha} f$ on $g$ is given by $\left(E^{-\alpha} f\right) g=E^{-\alpha}(f g), f, g \in \mathcal{B}$. The operator $X^{\prime} \dagger$ can be explained as an adjoint operator of $X^{\prime}$, if the inner products

$$
\langle Y, Z\rangle=\sum_{n \in \mathbb{Z}^{p}} \sum_{i=1}^{s} Y_{i}(n) Z_{i}(n), Y=\left(Y_{1}, \cdots, Y_{s}\right)^{T}, Z=\left(Z_{1} \cdots, Z_{s}\right)^{T} \in \mathcal{B}^{s}, s \geq 1,
$$

are well defined over some selected space of $u$. For example, for the adjoint operator $E_{i}^{\dagger}=E_{i}^{-1}$ of each shift operator $E_{i}(1 \leq i \leq p)$, we have

$$
\left\langle E_{i}^{\dagger} Y, Z\right\rangle=\left\langle E_{i}^{-1} Y, Z\right\rangle=\left\langle Y, E_{i} Z\right\rangle, Y, Z \in \mathcal{B} .
$$

Let us now consider an discrete evolution equation

$$
u_{t}=K(n, t, u), K \in \mathcal{A}^{q}
$$

its linearized equation and adjoint linearized equation are defined by

$$
\begin{aligned}
& (\sigma(n, t, u))_{t}=K^{\prime}(n, t, u) \sigma(n, t, u), \sigma \in \mathcal{B}^{q} \\
& (\rho(n, t, u))_{t}=-K^{\prime \dagger}(n, t, u) \rho(n, t, u), \rho \in \mathcal{B}^{q}
\end{aligned}
$$

respectively. Here $K^{\prime}$ and $K^{\prime \dagger}$ denotes the Gateaux derivative operator and the adjoint Gateaux derivative operator of $K$ with respect to $u$, respectively; and $f_{t}$ denotes the total derivative of $f$ with respect to $t$.

Definition 2.1. A vector field $\sigma \in \mathcal{B}^{q}$ is called a symmetry of the discrete evolution Equation (8), if it satisfies the linearized Equation (9) when u solves Equation (8). A vector field $\rho \in \mathcal{B}^{q}$ is called an adjoint symmetry of the discrete evolution equation Equation (8), if it satisfies the adjoint linearized Equation (10) when u solves (8). 
It is easy to see that two local vector fields $\sigma \in \mathcal{A}^{q}$ and $\rho \in \mathcal{A}^{q}$ are a symmetry and an adjoint symmetry of the discrete evolution Equation (8), if and only if they satisfy

$$
\begin{aligned}
& \frac{\partial \sigma(n, t, u)}{\partial t}=K^{\prime}(n, t, u) \sigma(n, t, u)-\sigma^{\prime}(n, t, u) K(n, t, u), \\
& \frac{\partial \rho(n, t, u)}{\partial t}=-K^{\prime \dagger}(n, t, u) \rho(n, t, u)-\rho^{\prime}(n, t, u) K(n, t, u),
\end{aligned}
$$

respectively, when $u$ solves Equation (8). Here $\sigma^{\prime}$ and $\rho^{\prime}$ are the Gateaux derivative operators of $\sigma$ and $\rho$, and $\frac{\partial}{\partial t}$ denotes the partial derivative with respect to $t$.

Definition 2.2. If a relation

$$
h_{t}=\sum_{i=1}^{p}\left(E_{i}-1\right) f_{i}, h, f_{i} \in \mathcal{B}, 1 \leq i \leq p,
$$

holds when $u$ solves the discrete evolution Equation (8), then Equation (13) is called a conservation law of Equation (8), $h$ a conserved density of Equation (8), and $f=\left(f_{1}, \cdots, f_{p}\right)^{T}$ a conversed flux of Equation (8) corresponding to $h$. A conserved density $h \in \mathcal{B}$ is called trivial, if there exist $g_{i} \in \mathcal{B}, 1 \leq$ $i \leq p$, such that $h=\sum_{i=1}^{p}\left(E_{i}-1\right) g_{i}$ holds on the solution set.

A conserved quantity means a quantity which does not vary with respect to time $t$ on the solution set. A quantity defined by $\mathcal{I}=\sum_{n \in \mathcal{Z}^{p}} I(n), I=I(n, t, u) \in \mathcal{B}$, is called a functional. We would first like to show a relation between conserved quantities and adjoint symmetries.

Proposition 2.1. Let $\mathcal{I}=\mathcal{I}(n, u)$ be a functional which does not depend explicitly on time $t$. Then, $\mathcal{I}$ is a conserved quantity of a discrete evolution equation $u_{t}=K, K=K(n, t, u) \in \mathcal{B}^{q}$, if and only if its variational derivative $\frac{\delta \mathcal{I}}{\delta u}$ is an adjoint symmetry of the same equation.

Proof: Let $\mathcal{S}$ be the Schwartz space and the Gateaux derivative of an object $P=P(u)$ with respect to $Y$ be defined by

$$
P^{\prime}[Y]=P^{\prime}(u)[Y]=\left.\frac{\partial P(n, t, u+\varepsilon Y)}{\partial \varepsilon}\right|_{\varepsilon=0}, Y \in \mathcal{B}^{q} .
$$

We denote the variational derivative $\frac{\delta \mathcal{I}}{\delta u}$ by $G$, and so, the definition of variational derivatives tells that

$$
\mathcal{I}^{\prime}[Y]=\langle G, Y\rangle, Y \in \mathcal{S}^{q}
$$

where the inner product is defined as in Equation (7). Then the differentiability of functions of $C^{\infty}$ class guarantees that on the solution set, we have

$$
\mathcal{I}_{t}=\frac{\partial \mathcal{I}}{\partial t}+\left.\frac{\partial \mathcal{I}\left(n, u+\varepsilon u_{t}\right)}{\partial \varepsilon}\right|_{\varepsilon=0}=\left\langle G, u_{t}\right\rangle=\langle G, K\rangle
$$

It now follows that

$$
\begin{aligned}
\left(\mathcal{I}_{t}\right)^{\prime}[Y] & =\left\langle G^{\prime}[Y], K\right\rangle+\left\langle G, K^{\prime}[Y]\right\rangle \\
& =\left\langle G^{\prime \dagger} K, Y\right\rangle+\left\langle K^{\prime \dagger} G, Y\right\rangle \\
& =\left\langle G^{\prime} K, Y\right\rangle+\left\langle K^{\prime \dagger} G, Y\right\rangle \\
& =\left\langle\frac{\partial G}{\partial t}+G^{\prime} K+K^{\prime \dagger} G, Y\right\rangle, Y \in \mathcal{S}^{q},
\end{aligned}
$$


where $G^{\prime \dagger}=G^{\prime}$ and $\frac{\partial G}{\partial t}=0$ were used. This last equality implies that $\mathcal{I}$ is conseved if and only if $G$ is an adjoint symmetry.

We use a conventional assumption below: an empty product of shift operators $E^{\alpha_{i}}, \alpha_{i} \in \mathbb{Z}, 1 \leq i \leq p$, is understood to be the identity operator. For example, the operator $\prod_{i=k}^{l} E_{i}^{2}$ implies the identity operator, when $k>l$. We would now like to prove the following lemma, in order to give a direct formula for constructing conservation laws of the discrete evolution Equation (8).

Lemma 2.1. Let $f$ and $g$ be two $C^{\infty}$ functions in variables $n_{1}, \cdots, n_{p}$. Then for any $\alpha=\left(\alpha_{1}, \cdots \alpha_{p}\right) \in \mathbb{Z}^{p}$, we have

$$
\begin{gathered}
f E^{\alpha} g-\left(E^{-\alpha} f\right) g=f\left(E_{1}^{\alpha_{1}} \cdots E_{p}^{\alpha_{p}} g\right)-\left(E_{1}^{-\alpha_{1}} \cdots E_{p}^{-\alpha_{p}} f\right) g \\
=\sum_{i=1}^{p}\left(E_{i}-1\right)\left[\sum_{\beta_{i}=1}^{\alpha_{i}}\left(E_{1}^{-\alpha_{1}} \cdots E_{i-1}^{-\alpha_{i-1}} E_{i}^{-\beta_{i}} f\right)\left(E_{i}^{\alpha_{i}-\beta_{i}} E_{i+1}^{\alpha_{i+1}} \cdots E_{p}^{\alpha_{p}} g\right)\right. \\
\left.-\sum_{\beta_{i}=1}^{-\alpha_{i}}\left(E_{1}^{-\alpha_{1}} \cdots E_{i-1}^{-\alpha_{i-1}} E_{i}^{-\alpha_{i}-\beta_{i}} f\right)\left(E_{i}^{-\beta_{i}} E_{i+1}^{\alpha_{i+1}} \cdots E_{p}^{\alpha_{p}} g\right)\right],
\end{gathered}
$$

where the value of an empty sum is conventionally zero.

Proof: First note that we have

$$
\begin{aligned}
& a E_{i}^{k} b-\left(E_{i}^{-k} a\right) b \\
= & \left(E_{i}-1\right)\left[\sum_{l=1}^{k}\left(E_{i}^{-l} a\right)\left(E_{i}^{k-l} b\right)-\sum_{l=1}^{-k}\left(E_{i}^{-k-l} a\right)\left(E_{i}^{-l} b\right)\right] \\
= & \begin{cases}\left(E_{i}-1\right) \sum_{l=1}^{k}\left(E_{i}^{-l} a\right)\left(E_{i}^{k-l} b\right), & k \geq 0, \\
\left(E_{i}-1\right) \sum_{l=1}^{-k}\left(E_{i}^{-k-l} a\right)\left(E_{i}^{-l} b\right), & k<0,\end{cases}
\end{aligned}
$$

for any $k \in \mathbb{Z}$ and any two $C^{\infty}$ functions $a$ and $b$ in variables $n_{1}, \cdots, n_{p}$. Then we decompose that

$$
\begin{aligned}
& f E^{\alpha} g-\left(E^{-\alpha} f\right) g=f\left(E_{1}^{\alpha_{1}} \cdots E_{p}^{\alpha_{p}} g\right)-\left(E_{1}^{-\alpha_{1}} \cdots E_{p}^{-\alpha_{p}} f\right) g \\
= & \sum_{i=1}^{p}\left[\left(E_{1}^{-\alpha_{1}} \cdots E_{i-1}^{-\alpha_{i-1}} f\right)\left(E_{i}^{\alpha_{i}} \cdots E_{p}^{\alpha_{p}} g\right)-\left(E_{1}^{-\alpha_{1}} \cdots E_{i}^{-\alpha_{i}} f\right)\left(E_{i+1}^{\alpha_{i+1}} \cdots E_{p}^{\alpha_{p}} g\right)\right] .
\end{aligned}
$$

It now follows from Equation (15) that each term in the above sum can be computed as follows:

$$
\begin{aligned}
& \left(E_{1}^{-\alpha_{1}} \cdots E_{i-1}^{-\alpha_{i-1}} f\right)\left(E_{i}^{\alpha_{i}} \cdots E_{p}^{\alpha_{p}} g\right)-\left(E_{1}^{-\alpha_{1}} \cdots E_{i}^{-\alpha_{i}} f\right)\left(E_{i+1}^{\alpha_{i+1}} \cdots E_{p}^{\alpha_{p}} g\right) \\
= & \left(E_{1}^{-\alpha_{1}} \cdots E_{i-1}^{-\alpha_{i-1}} f\right)\left[E_{i}^{\alpha_{i}}\left(E_{i+1}^{\alpha_{i+1}} \cdots E_{p}^{\alpha_{p}} g\right)\right] \\
& -\left[E_{i}^{-\alpha_{i}}\left(E_{1}^{-\alpha_{1}} \cdots E_{i-1}^{-\alpha_{i-1}} f\right)\right]\left(E_{i+1}^{\alpha_{i+1}} \cdots E_{p}^{\alpha_{p}} g\right) \\
= & \left(E_{i}-1\right)\left[\sum_{\beta_{i}=1}^{\alpha_{i}}\left(E_{1}^{-\alpha_{1}} \cdots E_{i-1}^{-\alpha_{i-1}} E_{i}^{-\beta_{i}} f\right)\left(E_{i}^{\alpha_{i}-\beta_{i}} E_{i+1}^{\alpha_{i+1}} \cdots E_{p}^{\alpha_{p}} g\right)\right. \\
& \left.-\sum_{\beta_{i}=1}^{-\alpha_{i}}\left(E_{1}^{-\alpha_{1}} \cdots E_{i-1}^{-\alpha_{i-1}} E_{i}^{-\alpha_{i}-\beta_{i}} f\right)\left(E_{i}^{-\beta_{i}} E_{i+1}^{\alpha_{i+1}} \cdots E_{p}^{\alpha_{p}} g\right)\right], 1 \leq i \leq p,
\end{aligned}
$$


where the value of an empty sum is conventionally zero. This allows us to conclude that the equality Equation (14) holds for any $\alpha=\left(\alpha_{1}, \cdots, \alpha_{p}\right) \in \mathbb{Z}^{p}$. The proof is finished.

Theorem 2.1. Let $\sigma=\left(\sigma_{1}, \cdots, \sigma_{q}\right)^{T} \in \mathcal{B}^{q}$ and $\rho=\left(\rho_{1}, \cdots, \rho_{q}\right)^{T} \in \mathcal{B}^{q}$ be a symmetry and an adjoint symmetry of the discrete evolution Equation (8), respectively. Then we have a conservation law of the discrete evolution Equation (8):

$$
\begin{aligned}
&\left(\sigma^{T} \rho\right)_{t}=\left(\sum_{i=1}^{q} \sigma_{i} \rho_{i}\right)_{t} \\
&=\sum_{k=1}^{p}\left(E_{k}-1\right) \sum_{i, j=1}^{q} \sum_{\alpha \in \mathbb{Z}^{p}}\left[\sum_{\beta_{k}=1}^{\alpha_{k}}\left(E_{1}^{-\alpha_{1}} \cdots E_{k-1}^{-\alpha_{k-1}} E_{k}^{-\beta_{k}} \rho_{i} \frac{\partial K_{i}}{\partial u_{\alpha}^{j}}\right)\left(E_{k}^{\alpha_{k}-\beta_{k}} E_{k+1}^{\alpha_{k+1}} \cdots E_{p}^{\alpha_{p}} \sigma_{j}\right)\right. \\
&\left.-\sum_{\beta_{k}=1}^{-\alpha_{k}}\left(E_{1}^{-\alpha_{1}} \cdots E_{k-1}^{-\alpha_{k-1}} E_{k}^{-\alpha_{k}-\beta_{k}} \rho_{i} \frac{\partial K_{i}}{\partial u_{\alpha}^{j}}\right)\left(E_{k}^{-\beta_{k}} E_{k+1}^{\alpha_{k+1}} \cdots E_{p}^{\alpha_{p}} \sigma_{j}\right)\right],
\end{aligned}
$$

where $\alpha=\left(\alpha_{1}, \cdots, \alpha_{p}\right)$, and the value of an empty sum is conventionally zero. Therefore, $\sigma^{T} \rho$ is a conserved density of the discrete evolution Equation (8).

Proof: Let us compute that

$$
\begin{aligned}
& \left(\sigma^{T} \rho\right)_{t}=\sigma_{t}^{T} \rho+\sigma^{T} \rho_{t}=\rho^{T} \sigma_{t}+\sigma^{T} \rho_{t} \\
= & \rho^{T} K^{\prime} \sigma-\sigma^{T} K^{\dagger \dagger} \rho=\sum_{i, j=1}^{q}\left(\rho_{i} V_{j}\left(K_{i}\right) \sigma_{j}-\sigma_{j} V_{j}^{\dagger}\left(K_{i}\right) \rho_{i}\right) \\
= & \sum_{i, j=1}^{q} \sum_{\alpha \in \mathbb{Z}^{p}}\left(\rho_{i} \frac{\partial K_{i}}{\partial u_{\alpha}^{j}} E^{\alpha} \sigma_{j}-\sigma_{j} E^{-\alpha} \rho_{i} \frac{\partial K_{i}}{\partial u_{\alpha}^{j}}\right) .
\end{aligned}
$$

By using Lemma 2.1, for all $1 \leq i, j \leq q, \alpha \in \mathbb{Z}^{p}$, we have

$$
\begin{aligned}
\rho_{i} \frac{\partial K_{i}}{\partial u_{\alpha}^{j}} E^{\alpha} \sigma_{j}-\sigma_{j} E^{-\alpha} \rho_{i} \frac{\partial K_{i}}{\partial u_{\alpha}^{j}} & \\
=\sum_{k=1}^{p}\left(E_{k}-1\right) & {\left[\sum_{\beta_{k}=1}^{\alpha_{k}}\left(E_{1}^{-\alpha_{1}} \cdots E_{k-1}^{-\alpha_{k-1}} E_{k}^{-\beta_{k}} \rho_{i} \frac{\partial K_{i}}{\partial u_{\alpha}^{j}}\right)\left(E_{k}^{\alpha_{k}-\beta_{k}} E_{k+1}^{\alpha_{k+1}} \cdots E_{p}^{\alpha_{p}} \sigma_{j}\right)\right.} \\
& \left.-\sum_{\beta_{k}=1}^{-\alpha_{k}}\left(E_{1}^{-\alpha_{1}} \cdots E_{k-1}^{-\alpha_{k-1}} E_{k}^{-\alpha_{k}-\beta_{k}} \rho_{i} \frac{\partial K_{i}}{\partial u_{\alpha}^{j}}\right)\left(E_{k}^{-\beta_{k}} E_{k+1}^{\alpha_{k+1}} \cdots E_{p}^{\alpha_{p}} \sigma_{j}\right)\right],
\end{aligned}
$$

where the value of an empty sum is conventionally zero. If then follows that the equality Equation (16) is true, and thus, $\sigma^{T} \rho$ is a conserved density of the discrete evolution Equation (8). The proof is finished.

This theorem tells us that a pair of a symmetry and an adjoint symmetry naturally yields a conservation law. Moreover, the expression of the conserved density is only dependent on the pair of a symmetry and an adjoint symmetry, but the expression of the conserved flux is dependent on the pair of a symmetry and an adjoint symmetry as well as the underlying equation.

If for two functions $h_{1}, h_{2} \in \mathcal{B}$, there exist functions $g_{i} \in \mathcal{B}, 1 \leq i \leq p$, such that

$$
h_{1}-h_{2}=\sum_{i=1}^{p}\left(E_{i}-1\right) g_{i}
$$


then we say that $h_{1}$ is equivalent to $h_{2}$, denoted by $h_{1} \sim h_{2}$. Obviously, this is an equivalence relation, and can be used in classifying conserved densities. A trivial conserved density is equivalent to zero.

\section{Applications to the Volterra Lattice Equation}

Now we go on to illustrate by an example rich structures of the conservation laws resulted from symmetries and adjoint symmetries.

Let us consider the Volterra lattice equation [8]:

$$
u_{t}=K(u)=u\left(E^{-1} u-E u\right), u=u(n, t), n \in \mathbb{Z}, t \in \mathbb{R} .
$$

Its linearized equation and adjoint linearized equation read

$$
\begin{aligned}
& \sigma_{t}=\left(E^{-1} u-E u\right) \sigma+u E^{-1} \sigma-u E \sigma, \\
& \rho_{t}=\left(E u-E^{-1} u\right) \rho-E(u \rho)+E^{-1}(u \rho),
\end{aligned}
$$

respectively. There exist infinitely many symmetries [9]:

$$
\begin{aligned}
& K_{i}=\Phi^{i}\left(u\left(E^{-1} u-E u\right)\right), i \geq 0, \\
& \tau_{i}=\Phi^{i}\left(t\left[K_{0}, u\right]+u\right)=\Phi^{i}\left(t K_{0}+u\right)=\Phi^{i}\left(t u\left(E^{-1} u-E u\right)+u\right), i \geq 0,
\end{aligned}
$$

where the hereditary recursion operator $\Phi$ is defined by

$$
\Phi=u\left(1+E^{-1}\right)\left(-(E u) E^{2}+u\right)(E-1)^{-1} u^{-1} .
$$

Moreover, these symmetries constitute a Lie algebra [9]:

$$
\left[K_{i}, K_{i}\right]=0,\left[K_{i}, \tau_{j}\right]=(i+1) K_{i+j},\left[\tau_{i}, \tau_{j}\right]=(i-j) \tau_{i+j}, i, j \geq 0
$$

where $[K, S]$ is defined by $[K, S]=K^{\prime} S-S^{\prime} K, K^{\prime}$ and $S^{\prime}$ being the corresponding Gateaux derivative operators.

By an inspection, the function defined by

$$
S_{0}=u^{-1}
$$

is an adjoint symmetry of the Volterra lattice Equation (18). This means that $S_{0}$ satisfies the adjoint linearized Equation (20) while $u$ solves Equation (18). The proof just needs a simple and direct computation:

$$
\left(S_{0}\right)_{t}=-u^{-2} u_{t},\left(E u-E^{-1} u\right) S_{0}-E\left(u S_{0}\right)+E^{-1}\left(u S_{0}\right)=\left(E u-E^{-1} u\right) u^{-1},
$$

from which it directly follows that if $u$ solves Equation (18), $S_{0}$ satisfies Equation (20). Now, using the principle in Theorem 2.1, we have infinitely many conserved densities, $u^{-1} K_{i}$ and $u^{-1} \tau_{i}, i \geq 0$. In particular, since we have

$$
\begin{aligned}
& S_{0} \tau_{1}=S_{0}\left(t K_{1}+\Phi u\right) \sim u^{-1} \Phi u, \\
& (\Phi u)(n)=u(n)[-(n+2) u(n+1)-u(n)+(n-1) u(n-1)],
\end{aligned}
$$


we obtain a nontrivial local conserved density of the Volterra lattice Equation (18):

$$
\left(h_{1}\right)(n)=-(n+2) u(n+1)-u(n)+(n-1) u(n-1) .
$$

This generates a conservation law

$$
h_{1 t}=(E-1)\left[([n]+1) u(E u)-([n]-1)\left(E^{-2} u\right)\left(E^{-1} u\right)\right],
$$

where $[n]$ is the operator

$$
([n] f)(m)=m f(m), f \in \mathcal{B}, m \in \mathbb{Z} .
$$

Except $S_{0} \tau_{1}=S_{0}\left(t K_{0}+u\right) \sim 1$, all other products of the same type, $u^{-1} \tau_{i}, i \geq 2$, give us nontrivial nonlocal conserved densities of the Volterra lattice Equation (18). But all $u^{-1} K_{i}, i \geq 0$, are trivial conserved densities, which can be seen directly from the recursion structure of symmetries [10].

Based on Proposition 2.1, the Hamiltonian formulation of the Volterra soliton hierarchy [9] guarantees that the Volterra lattice Equation (18) has a hierarchy of adjoint symmetries:

$$
\rho_{i}=\Psi^{i} 1=\frac{\delta \mathcal{H}_{i}}{\delta u}, \Psi=\Phi^{\dagger}=u^{-1}(E-1)^{-1}\left(-(E u) E^{2}+u\right)\left(1+E^{-1}\right) u, i \geq 0,
$$

which generate, by Theorem 2.1, the conserved densities: $\rho_{i} K_{j}, \rho_{i} \tau_{j}, i, j \geq 0$. The nontrivial conserved density generated from $\rho_{0} \tau_{1}$ is, due to $K_{1} \sim 0$, equivalent to

$$
\left(h_{2}\right)(n)=(\Phi u)(n)=u(n)[-(n+2) u(n+1)-u(n)+(n-1) u(n-1)],
$$

whose associated conservation law reads

$$
h_{2 t}=(E-1)\left\{([n]+1)\left[\left(E^{-1} u\right) u^{2}+\left(E^{-1} u\right) u(E u)\right]-([n]-1)\left[\left(E^{-2} u\right)^{2} u+\left(E^{-2} u\right)\left(E^{-1} u\right) u\right]\right\} .
$$

Moreover, we see that all symmetries, $K_{i}=\rho_{0} K_{i}$ and $\tau_{i}=\rho_{0} \tau_{i}, i \geq 0$, are also conserved densities, and that the conserved densities $\rho_{i} \tau_{0} \sim \rho_{i} u, i \geq 0$, present all standard ones associated with the Hamiltonian functionals $\left\{\mathcal{H}_{i} \mid i \geq 0\right\}$ [9], upon noting

$$
u \frac{\delta \mathcal{H}}{\delta u}=u \sum_{\alpha \in \mathcal{Z}} E^{-\alpha} \frac{\partial H}{\partial\left(E^{\alpha} u\right)} \sim \sum_{\alpha \in \mathcal{Z}}\left(E^{\alpha} u\right) \frac{\partial H}{\partial\left(E^{\alpha} u\right)}=H, \mathcal{H}=\sum_{n \in \mathcal{Z}} H(n), H \in \mathcal{A} .
$$

The Volterra lattice Equation (18) also has the following Lax pair [11]:

$$
U(u, \lambda)=\left[\begin{array}{cc}
0 & u \\
-1 & \lambda
\end{array}\right], V(u, \lambda)=\left[\begin{array}{cc}
\lambda^{2}-u & -\lambda E^{-1} u \\
\lambda & -E^{-1} u
\end{array}\right]
$$

which means that the Volterra lattice Equation (18) is equivalent to the discrete zero curvature equation $U_{t}=(E V) U-U V$. Let $\lambda_{s}, 1 \leq s \leq N$, be arbitrary constants, and introduce $N$ replicas of the spectral problems:

$$
E \phi^{(s)}=U\left(u, \lambda_{s}\right) \phi^{(s)}, \phi_{t}^{(s)}=V\left(u, \lambda_{s}\right) \phi^{(s)}, \phi^{(s)}=\left(\phi_{1 s}, \phi_{2 s}\right)^{T}, 1 \leq s \leq N,
$$

and $N$ replicas of the adjoint spectral problems:

$$
E \psi^{(s)}=\left(U^{T}\right)^{-1}\left(u, \lambda_{s}\right) \psi^{(s)}, \psi_{t}^{(s)}=-V^{T}\left(u, \lambda_{s}\right) \psi^{(s)}, \psi^{(s)}=\left(\psi_{1 s}, \psi_{2 s}\right)^{T}, 1 \leq s \leq N .
$$


Then, we have a class of adjoint symmetries represented in terms of eigenfunctions and adjoint eigenfunctions [11]:

$$
T_{0}=\frac{1}{u}\left(P_{2}^{T} A Q_{1}+P_{2}^{T} Q_{2}\right)
$$

where the matrix $A$, and the two $N$ dimensional vector functions $P_{i}$ and $Q_{i}$ are defined by

$$
A=\operatorname{diag}\left(\lambda_{1}, \cdots, \lambda_{N}\right), P_{i}=\left(\phi_{i 1}, \cdots, \phi_{i N}\right)^{T}, Q_{i}=\left(\psi_{i 1}, \cdots, \psi_{i N}\right)^{T}, 1 \leq i \leq 2 .
$$

Now, Theorem 2.1 guarantees that we have the conserved densities: $T_{0} K_{i}$ and $T_{0} \tau_{i}, i \geq 0$. This particularly gives the following two conserved densities:

$$
\begin{aligned}
& T_{0} K_{0}=\left(E^{-1} u-E u\right)\left(P_{2}^{T} A Q_{1}+P_{2}^{T} Q_{2}\right), \\
& T_{0} \tau_{0}=t\left(E^{-1} u-E u\right)\left(P_{2}^{T} A Q_{1}+P_{2}^{T} Q_{2}\right)+\left(P_{2}^{T} A Q_{1}+P_{2}^{T} Q_{2}\right) .
\end{aligned}
$$

Again by Theorem 2.1, their corresponding fluxes read

$$
\begin{aligned}
& -\left(P_{2}^{T} A Q_{1}+P_{2}^{T} Q_{2}\right) E^{-1} K_{0}-\left(E\left(P_{2}^{T} A Q_{1}+P_{2}^{T} Q_{2}\right)\right) K_{0} \\
& -\left(P_{2}^{T} A Q_{1}+P_{2}^{T} Q_{2}\right) E^{-1} \tau_{0}-\left(E\left(P_{2}^{T} A Q_{1}+P_{2}^{T} Q_{2}\right)\right) \tau_{0}
\end{aligned}
$$

respectively. The involvement of eigenfunctions and adjoint eigenfunctions exhibits strong nonlocality of this kind of conservation laws.

\section{Concluding Remarks}

We have established a direct approach for constructing conservation laws and thus conserved densities of discrete evolution equations, whether the evolution equations are derivable from a Lagrangian or not. Our approach utilizes pairs of symmetries and adjoint symmetries, in which adjoint symmetries make up for the disadvantage of non-Lagrangian structures in establishing a correspondence between symmetries and conservation laws. The approach has been applied to the generation of conserved densities of the Volterra lattice equation.

We remark that on one hand, all evolution equations become the first-order ordinary differential equations (ODEs), when there is no spatial shift appeared in the equations. On the other hand, if we remove the time derivative, i.e., there is no time involved in evolution equations, then we immediately obtain conservation laws for difference equations. Moreover, our results pave a way to construct conservation laws from symmetries for self-adjoint discrete evolution equations or difference equations, since symmetries are also adjoint symmetries of self-adjoint discrete evolution equations or difference equations. We also point out that our idea of constructing conservation laws by symmetries and adjoint symmetries is quite similar to that of carrying out binary nonlinearization under symmetry constraints [12,13]. In the theory of binary nonlinearization [12,13], adjoint symmetries are used to establish a balance with non-Lie adjoint symmetries generated from both spectral problems and adjoint spectral problems.

There are many other approaches or theories on conservation laws of differential equations and differential-difference equations. In the case of integrable equations, Hamiltonian formulations [14,15] generate usual conservation laws associated with Hamiltonian functionals, and a bi-Hamiltonian 
formulation presents a recurrence relation of conserved densities in the resulting conservation laws [16]. Moreover, Lax pairs are used to make Riccati type equations that ratios of eigenfunctions need to satisfy, and series expansions of solutions of the resulting Riccati type equations around the spectral parameter yield conservation laws (see, e.g., [17,18]). For continuous evolution equations, adjoint symmetries are also called conserved covariants [19], and it is recognized that the product of a symmetry and an adjoint symmetry presents a conserved density (see, e.g., [19-21]) and that a functional $\mathcal{I}$ is conserved if and only if its variational derivative $\frac{\delta \mathcal{I}}{\delta u}$ is an adjoint symmetry (see, e.g., [13,20,21]).

There exists a geometrical theory to deal with adjoint symmetries of the second-order ODEs [22]. Adjoint symmetries are also used to show separability of finite-dimensional Hamiltonian systems (see, e.g., [23]) and links to integrating factors of the second-order ODEs (see, e.g., [24]). An important character for symmetries is the existence of Lie algebraic structures, and such Lie algebraic structures can be resulted from a kind of Lie algebras of Lax operators corresponding to symmetries (see [25-27] for the continuous case and [9,28] for the discrete case). Therefore, we are curious about whether there exist any Lie algebraic structures for adjoint symmetries and what kind of Lie algebraic structures we can have if they exist. We finally make a remark about this question. A natural binary operation for adjoint symmetries could be taken as

$$
\llbracket \rho_{1}, \rho_{2} \rrbracket=\left(\rho_{1}^{\prime}\right)^{\dagger} \rho_{2}-\left(\rho_{2}^{\prime}\right)^{\dagger} \rho_{1},
$$

where $\left(\rho_{1}^{\prime}\right)^{\dagger}$ and $\left(\rho_{2}^{\prime}\right)^{\dagger}$ denote their adjoint Gateaux derivative operators. However, this doesn't keep the space of adjoint symmetries closed. For example, for the Volterra lattice Equation (18), we have two adjoint symmetries $\rho_{1}=1$ and $\rho_{2}=u^{-1}$, which generate

$$
\llbracket \rho_{1}, \rho_{2} \rrbracket=\llbracket 1, u^{-1} \rrbracket=u^{-2}
$$

but this function $u^{-2}$ is not an adjoint symmetry of the Volterra lattice equation.

\section{Acknowledgments}

The work was supported in part by NNSFC under the grants 11371326, 11271008 and 1371361, the Fundamental Research Funds for the Central Universities (2013XK03), the Natural Science Foundation of Shandong Province (Grant No. ZR2013AL016), Zhejiang Innovation Project of China (Grant No. T200905), the First-class Discipline of Universities in Shanghai and the Shanghai University Leading Academic Discipline Project (No. A.13-0101-12-004), and Shanghai University of Electric Power.

\section{Conflicts of Interest}

The author declares no conflict of interest.

\section{References}

1. Bluman, G.; Kumei, S. Symmetries and Differential Equations; Springer-Verlag: New York, NY, USA, 1989. 
2. Olver, P.J. Applications of Lie Groups to Differential Equations; Graduate Texts in Mathematics 107; Springer-Verlag: New York, NY, USA, 1993.

3. Ibragimov, N.H. Transformation Groups Applied to Mathematical Physics; Mathematics and its Applications (Soviet Series); D. Reidel Publishing Co.: Dordrecht, The Netherlands, 1985.

4. Morawetz, C.S. Variations on Conservation Laws for the Wave Equation. Bull. Amer. Math. Soc. 2000, 37, 141-154.

5. Anco, S.C.; Bluman, G. Direct Computation of Conservation Laws from Field Equations. Phys. Rev. Lett. 1997, 78, 2869-2873.

6. Ibragimov, N.H. A New Conservation Theorem. J. Math. Anal. Appl. 2007, 333, 311-328.

7. Ibragimov, N.H. Nonlinear Self-Adjointness and Conservation Laws. J. Phys. A Math. Theor. 2011, 44, doi:10.1088/1751-8113/44/43/432002.

8. Volterra, V. Leçons sur la Théorie Mathématique de la Lutte pour la vie; Gauthier-Villars: Paris, France, 1931. (In French)

9. Ma, W.X.; Fuchssteiner, B. Algebraic Structure of Discrete Zero Curvature Equations and Master Symmetries of Discrete Evolution Equations. J. Math. Phys. 1999, 40, 2400-2418.

10. Fuchssteiner, B.; Ma, W.X. An Approach to Master Symmetries of Lattice Equations. In Symmetries and Integrability of Difference Equations; Clarkson, P.A., Nijhoff, F.W., Eds.; London Mathematical Society Lecture Note Series 255; Cambridge University Press: Cambridge, UK, 1999; pp. 247-260.

11. Ma, W.X.; Geng, X.G. Bäcklund Transformations of Soliton Systems from Symmetry Constraints. In Bäcklund and Darboux Transformations-The Geometry of Solitons; CRM Proceedings and Lecture Notes. 29; American Mathematical Society: Providence, RI, USA, 2001; pp. 313-323.

12. Ma, W.X.; Strampp, W. An Explicit Symmetry Constraint for the Lax Pairs and the Adjoint Lax Pairs of AKNS Systems. Phys. Lett. A 1994, 185, 277-286.

13. Ma, W.X.; Zhou, R.G. Adjoint Symmetry Constraints Leading to Binary Nonlinearization. J. Nonlinear Math. Phys. 2002, 9, 106-126.

14. Tu, G.Z. On Liouville Integrability of Zero-Curvature Equations and the Yang Hierarchy. J. Phys. A Math. Gen. 1989, 22, 2375-2392.

15. Ma, W.X.; Chen, M. Hamiltonian and Quasi-Hamiltonian Structures Associated with Semi-Direct Sums of Lie Algebras. J. Phys. A Math. Gen. 2006, 39, 10787-10801.

16. Magri, F. A Simple Model of the Integrable Hamiltonian Equation. J. Math. Phys. 1978, 19, 1156-1162.

17. Alberty, J.M.; Koikawa, T.; Sasaki, R. Canonical Structure of Soliton Equations I. Phys. D Nonlinear Phenom. 1982, 5, 43-65.

18. Zhang, D.J.; Chen, D.Y. The Conservation Laws of Some Discrete Soliton Systems. Chaos Solitons Fractals 2002, 14, 573-579.

19. Fuchssteiner, B.; Fokas, A.C. Symplectic Structures, Their Bäcklund Transformations and Hereditary Symmetries. Phys. D Nonlinear Phenom. 1981, 4, 47-66.

20. Tu, G.Z.; Qin, M.Z. Relationship Between Symmetries and Conservation Laws of Nonlinear Evolution Equations. Chin. Sci. Bull. 1979, 24, 913-917. (In Chinese) 
21. Fu, W.; Huang, L.; Tamizhmani, K.M.; Zhang, D.J. Integrability Properties of the Differential-Difference Kadomtsev-Petviashvili Hierarchy and Continuum Limits. Nonlinearity 2013, 26, 3197-3229.

22. Morando, P.; Pasquero, S. The Symmetry in the Structure of Dynamical and Adjoint Symmetries of Second-Order Differential Equations. J. Phys. A Math. Gen. 1995, 28, 1943-1955.

23. Sarlet, W.; Ramos, A. Adjoint Symmetries, Separability, and Volume Forms. J. Math. Phys. 2000, 41, 2877-2888.

24. Mohanasubha, R.; Chandrasekar, V.K.; Senthilvelan, M.; Lakshmanan, M. Interplay of Symmetries, Null Forms, Darboux Polynomials, Integrating Factors and Jacobi Multipliers in Integrable Second-Order Differential Equations. Proc. R. Soc. Lond. Ser. A Math. Phys. Eng. Sci. 2014, 470, doi:10.1098/rspa.2013.0656.

25. Ma, W.X. The Algebraic Structures of Isospectral Lax Operators and Applications to Integrable Equations. J. Phys. A Math. Gen. 1992, 25, 5329-5343.

26. Ma, W.X. Lax Representations and Lax Operator Algebras of Isospectral and Nonisospectral Hierarchies of Evolution Equations. J. Math. Phys. 1992, 33, 2464-2476.

27. Ma, W.X. Lie Algebra Structures Associated with Zero Curvature Equations and Generalized Zero Curvature Equations. Br. J. Appl. Sci. Tech. 2013, 3, 1336-1344.

28. Ma, W.X. A Method of Zero Curvature Representation for Constructing Symmetry Algebras of Integrable Systems. In Proceedings of the 21st International Conference on the Differential Geometry Methods in Theoretical Physics; Ge, M.L., Ed.; World Scientific: Singapore, 1993; pp. 535-538.

(c) 2015 by the author; licensee MDPI, Basel, Switzerland. This article is an open access article distributed under the terms and conditions of the Creative Commons Attribution license (http://creativecommons.org/licenses/by/4.0/). 